\title{
Langue et littérature hébraïques médiévales et modernes en Occident
}

Jean-Pierre Rothschild

\section{OpenEdition}

\section{Journals}

Édition électronique

URL : https://journals.openedition.org/ashp/1137

DOI : $10.4000 /$ ashp. 1137

ISSN : 1969-6310

Éditeur

Publications de l'École Pratique des Hautes Études

\section{Édition imprimée}

Date de publication : 1 octobre 2011

Pagination : $32-34$

ISSN : 0766-0677

\section{Référence électronique}

Jean-Pierre Rothschild "Lanque et littérature hébraïques médiévales et modernes en Occident »,

Annuaire de l'École pratique des hautes études (EPHE), Section des sciences historiques et philologiques

[En ligne], 142 | 2011, mis en ligne le 21 juillet 2011, consulté le 06 juillet 2021. URL : http://

journals.openedition.org/ashp/1137; DOI : https://doi.org/10.4000/ashp.1137 


\title{
LANGUE ET LITTÉRATURE HÉBRAÏQUES MÉDIÉVALES ET MODERNES EN OCCIDENT
}

\author{
Directeur d'études : M. Jean-Pierre RoTHSCHILD
}

Programme de l'année 2009-2010 : I. Le Livre des éléments d'Isaac Israéli et ses traductions latines. - II. Réception de l'Éthique à Nicomaque dans la littérature hébraïque en Espagne au $X V^{e}$ siècle.

\section{Le Livre des éléments d'Isaac Israéli et ses traductions latines}

En l'absence d'auditeurs qui fussent aussi des latinistes confirmés, nous avons peu poursuivi cette année le travail, entrepris l'an dernier, d'établissement du texte des deux versions latines que le directeur d'études avait naguère distinguées du Livre des éléments d'Isaac Israéli. Il n'entrait pas dans nos plans de reprendre pour elle seule la réédition des deux versions hébraïques éditées conjointement par S. Fried en 1900 et nous ne disposions pas encore de tous les manuscrits requis pour conduire sur ce point un travail de fond. Nous nous sommes donc limités cette année à quelques lectures tant dans les textes latins qu'hébreux et à des rappels de données cosmologiques de base de la théorie élémentaire.

\section{Réception de l'Éthique à Nicomaque dans la littérature hébraïque en Espagne au $X V^{e}$ siècle}

L'essentiel du travail de l'année a donc porté sur la réception espagnole de l'Éthique en hébreu, plus précisément, sur les deux abrégés du texte d'Aristote rédigés indépendamment par Joseph b. Shem Tov ibn Shem Tov et par son fils Shem Tov ben Joseph. En quatre générations, Rabbi Nissim de Gérone ( ca 1320-1380) et ceux qui ont repris ses ensignements jusqu'à son arrière arrière-disciple Isaac Abrabanel (1437-1508) ont accommodé en hébreu l'Éthique à Nicomaque, dont il ne leur échappait pas que l'universalité comportait une mise en question théorique et un péril pratique pour la spécificité juive, et ont limité la portée de sa réception, chacun selon sa stratégie particulière. La découverte tardive du texte même de l'Éthique n'avait pas été une révélation : Maïmonide (1135/8-1204), en particulier dans son introduction au traité de la Michna dit Traité des Principes ou Maximes des Pères ( $\breve{S}^{e} m \bar{m} n \bar{a} h p^{e}$ rāqūm, Huit chapitres) et dans son code légal Mišnēh Tōrāh, sous les règles regardant les rois (Hilekhōt $m^{e} l a \bar{k} h \bar{i} m$ ), en avait laissé paraître beaucoup sans, probablement, avoir disposé lui-mêrme du texte d'Aristote, l'ayant connu à travers des florilèges, des lieux communs, des références des auteurs arabes. De plus, ce qui en passait dans des instruments du même type au temps des auteurs considérés et ce qui, plus insaisissable encore, relève de « l'air 
du temps ${ }^{1}$ » avait-il prépéré sa réception ${ }^{2}$. La traduction de l'arabe du Commentaire Moyen ou praphrase d'Averroès à l'Éthique par Samuel b. Juda de Marseille faite en Provence au début des années 1320 donne un accès plus direct au texte lui-même. Enfin il est traduit du latin par Meïr Alguadez en Espagne vers 1400, désormais dégagé de la gangue du commentateur mais par là plus difficile encore, d'où plusieurs commentaires hébreux : l'un d'Alguadez lui-même, conservé dans le seul manuscrit Madrid, BN 5459; un autre, plus répandu, mis sous le nom de saint Thomas, accompagnant le texte du Commentaire Moyen d'Averroès traduit par Samuel de Marseille, apparemment pour réunir les deux traditions, arabe et latine, d'interprétation de l'ouvrage; celui de Joseph ibn Shem Tov (1442); celui de Barukh Ibn Ya'iš (vers 1485). La plupart de ces textes sont pourvus d'introductions éclairantes quant à leurs motifs et leurs méthodes, que le directeur d'études a étudiées ailleurs.

Le manuscrit de Paris, BN(F), hébreu 892, d'une écriture espagnole datable au $\mathrm{XV}^{\mathrm{e}}$ siècle, donc très proche dans le temps de la composition des textes eux-mêmes, est un recueil homogène, incomplet, consacré à la philosophie morale. Il comporte aux f. 1-85v la traduction de l'Éthique par Meïr Alguadez; aux f. 85v-90v, celles des Économiques (pseudo-)aristotéliciens; aux f. 91-115, une table de l'Éthique (Mafteah sefer ha-middot... u-feyrush qasar nora' me'od, « Table de l'Éthique et commentaire court et très remarquable ») attribuée à Joseph b. Shem Tov; aux f. 116-133, un florilège de l'Éthique par Shem Tov [b. Joseph] Ben-Shem Tov; aux f. 134-135v, un florilège des M'ozney sedeq d'Al-Gâzâlî, seul texte qui ne soit pas d'Aristote, bien qu'il y fasse référence, ouvrage arabe d'éthique traduit en hébreu dès le XIII ${ }^{\mathrm{e}}$ siècle par Abraham ibn Ḥasdaï (début seulement; le manuscrit est mutilé).

Après avoir défini les enjeux de la réception de l'Éthique parmi les philosophes juifs d'Espagne en général et dans l'école issue de Nissim de Gérone en particulier, nous nous sommes attachés à déterminer les rapports entre la table et le florilège des ibn Shem Tov et les précédents latins les plus connus, la Sententia libri Ethicorum et la Tabula libri Ethicorum de saint Thomas d'Aquin (lus dans l'édition léonine, t. XLVII, 1969 et XLVIII, 1971), puis à comparer la méthode et la teneur de la table de Joseph b. Shem Tov avec le florilège de son fils Shem Tov ben Joseph. En effet, ainsi que le directeur d'études l'a établi il y a quelques années ${ }^{3}$, autant il est vrai que Shem Tov b. Joseph est un disciple fidèle de l'école rationaliste issue de Maïmonide - son commentaire du Guide des égarés est d'ailleurs devenu un classique entré dans les éditions courantes de la traduction hébraïque de cet ouvrage - autant son père, en

1. L. Sturlese, in Ulrich von Strassburg, De summo bono, Liber 4, Tractatus 2, 15-24, éd. B. Mojsisch et F. Retucci, Hambourg, 2008, p. IX : « Es geht hierbei nicht allein um positiv nachweisbare Intertextualität, sondern um Momente einer allgemeineren, nur schwer genau festzumachenden Interdiskursivität, die aus bestimmten Schlagworten, aus spezifischen Themen, aus gemeinsamen Fragen, aus verwandten Begriffen und Anspielungen besteht $»$.

2. Un tel processus a été bien décrit pour un autre texte de philosophie morale d'Aristote : Fr. Bertelloni, «Presupuestos de la recepción de la Politica de Aristóteles », dans F. Domínguez, R. Imbach, Th. Pindl et P. Walter (éd.), Aristotelica et Lulliana magistro doctissimo Charles H. Lohr septuagesinum annum feliciter agenti dedicata, Steenbruge, 1995, p. 35-54, « processus préparatoire » à la réception qui débute v. 1150 et prend fin avec la traduction effective.

3. J.-P. Rothschild, « Le dessein philosophique de Joseph Ibn Shem Tob (Espagne, flor. 1442-1455) », Revue des études juives, 162/ 1-2 (janvier-juin 2003), p. 97-122. 
dépit de certaines apparences, se révèle un critique systématique de cette école, se plaçant lui-même dans la filière de Nissim de Gérone et du grand critique d'Aristote Hasdaï Crescas, dont il fut le traducteur et probablement l'élève. En un premier temps, l'un et l'autre instruments, qui suivent l'ordre du texte d'Aristote, apparaissent comme des abrégés de l'Éthique fidèles à la lettre du Stagyrite et des différences de positions philosophiques, tant l'un par rapport à l'autre que par rapport à Aristote, ne se relèvent pas aisément. Il faudra attendre pour tirer des conclusions d'avoir pris une vue plus complète des deux ouvrages. Quant à la comparaison avec Thomas d'Aquin, elle ne se fait pas sans transposition, la Sententia étant un commentaire bien plus étendu que les abrégés hébreux et la Tabula étant alphabétique, donc rompant la linéarité du texte d'Aristote respectée au contraire par les Shem Tov. Il n'est pas exclu qu'aient pu être utilisés des commentateurs latins de l'Éthique moins célèbres mais plus proches dans le temps et dans l'espace comme Pierre de Osma (ob. 1480), professeur à l'université de Salamanque, ou Jean Versor ( $o b$. 1477), maître parisien qui semble avoir séjourné en Aragon (d'après la chronique hébraïque du $\mathrm{XvI}^{\mathrm{e}}$ s. Shebet Yehuda de Salomon ibn Varga) et dont plusieurs ouvrages furent traduits en hébreu par 'Eli Habilio, lequel entretint des relations avec Shem Tov b. Joseph. La recherche de l'usage possible de ces commentaires sera à mener ultérieurement. 